\title{
Analyzing the Problems in Managing Patients with Medically Unexplained Symptoms
}

\author{
Winfried Rief, $P h D$ \\ Clinical Psychology and Psychotherapy, Philipps University of Marburg, Gutenbergstrasse 18, Marburg 35032, Germany.
}

DOI: $10.1007 / \mathrm{s} 11606-007-0151-2$

(C) 2007 Society of General Internal Medicine 2007;22:704-706 the overwhelming number of nearly 300 mental disorder diagnoses in DSM-IV, a complexity that cannot be managed in everyday practice, and which has to be reduced to major clusters.

These patients are difficult (but not all of them). It is an unspoken assumption in medicine that patients present medical problems if they have medical diseases, and psychological problems if they have mental disorders. In the area of MUS, this is by definition not the case. Even in psychiatric conditions such as depression or anxiety disorders, most patients present to the doctor with physical complaints rather than psychological symptoms. ${ }^{9-11}$ It is not only the presentation of somatic complaints, but also the inflexibility of patients in never abandoning their search for medical explanations, which is thought to contribute to the problem. However, while some of these patients really do have rigid attitudes about their symptoms, the majority of patients with MUS do accept multiple explanations for their symptoms, even though they present just one explanation to their doctor. ${ }^{12}$ The challenge for the doctor is to detect patient's additional interpretations about the origin of their symptoms that might be more helpful.

A major source of misunderstandings between doctors and patients are biases in patient's information processing. In a recent study, we found that even when doctors explained that some medical conditions were unlikely to be the cause of the symptoms, patients recalled an overly high likelihood of these diseases. ${ }^{13}$ Erroneous remembered medical information is not only a source of misunderstandings and worsening of the doctor-patient relationship, but also a critical predictor of poor course and outcome. A further challenge in the medical encounter is that many patients with MUS tend to be suspicious and to mistrust doctors' information. It is still unclear whether this mistrust is a personality trait of these patients, or whether it is a result of former negative experiences in medical encounters. Successful management of patients with MUS has to address the subjective illness perceptions, possibly underlying illness fears and information-processing biases. However, this requires not only knowledge about the patient, but also behavioral skills in the doctor.

Guidelines for managing the MUS problem. Most patients with MUS seek medical help in family and Internal Medicine offices. ${ }^{10,14}$ This points to the central role of this profession in finding the right way to manage these patients. Physicians need guidelines that are feasible in the short period of interaction between the patient and doctor. These guidelines should not only include medical decisions about investigations and 


\section{Table 1. Examples of Behavioral Guidelines for Doctors Treating MUS $^{7,13,15}$}

Confirm the credibility of the patient's complaints.

Early on in treatment, address the fact that the most likely cause of the patient's complaints is not a severe organic illness but rather a disorder of his/her perception of body processes or stress symptom.

Explore physical and possible psychological symptoms as completely as possible.

Discuss the planned examinations and their consequences with the patient as early as possible. Anticipate when you will stop with medical investigations.

Avoid unnecessary medical investigations and petty diagnoses.

Arrange fixed appointments for follow-up examinations.

Motivate patients to lead their lives healthily, to reduce stress, and to get a sufficient amount of physical exercise. Prevent inadequate avoidance behavior.

Ask patients to give summaries of what you told them to detect possible information processing biases or misunderstandings.

therapies, but also behavioral recommendations about how to interact with patients with MUS. These recommendations should include: how to explain to a patient that no serious medical condition is causing the symptoms, even though the patient is suffering tremendously; how to react if medical reassurance is failing; and how to deal with a patient's pressure for further medical investigations if the doctor is sure that this is not necessary or even harmful? Some examples for these guidelines are already published (Table 1). ${ }^{15}$ However, most studies offering trainings for family and Internal Medicine physicians on how to manage patients with MUS reported tremendously low rates of participation. In one of our own studies, less than $10 \%$ of physicians who were invited to participate in a 1-day training program actually attended, even though participants received $€ 500$ (U.S. \$600) compensation. Therefore, the analysis by Salmon et al. ${ }^{16}$ about why general practitioners decline training to improve management of MUS is of central relevance.

One of the major tasks for a physician is to decide which diagnostic tests to perform to balance attentiveness and overinvestigation. The risk is that patients' psychological distress and complaining behavior can influence the doctor's decisions about medical investigations and treatments. Patients who complain more receive more medical investigations. As this relationship can be problematic, doctors should be trained to disentangle these influences on the medical decision process.

Which symptoms are medically unfounded? A final problem is that the decision about the etiology of physical complaints is highly dependent on the personality of the physician. Physicians' estimations of how many patients in their offices have MUS are extremely varying, although psychometric ratings show highly comparable prevalence rates in different offices. ${ }^{1}$ Every doctor has his own rating system for which kind of symptoms are medically explained versus medically unexplained. As long as the problem of etiology assumptions is not solved, all classification approaches for MUS do not lead to reliable results.

Medical education. Most physicians are well trained in detecting medical conditions: knowing about their biological backgrounds and choosing the appropriate treatment if available. By means of medical education, and by legal regulations, physicians are biased to focus on potentially hidden diseases. While these conditions are typically less frequent, MUS are a major part of practitioners' everyday life and represent $20-40 \%$ of patients. Therefore, physicians must be adequately trained to manage this large group of patients with MUS. In most countries, the high relevance of MUS for everyday practice is not reflected in curricula of medical education.

For the management of patients with MUS, physicians do not only need technical equipment, but verbal and behavioral skills as described above. These behavioral skills should be taught in medical education programs by role-plays and other techniques that give participants the opportunity to practice new skills.

The medicolegal system. Most medicolegal systems have some regulations making it difficult to manage this patient group adequately. Gratification is offered to patients in many ways for the expression of physical complaints, but less for the expression of psychological distress. Physicians are legally threatened when overlooking medical conditions, but do not experience any negative consequences if their management strategies of patients with MUS are inappropriate, or if they overinvestigate these patients. It is an urgent need to develop health care systems that offer reinforcement for those doctors using adequate management principles for patients with MUS.

\section{CONCLUSION}

It is clear that many steps need to be taken to improve the management of patients with MUS. It is not only having the knowledge about the patients and pathophysiological processes, but it is also a modification in the behavioral skills of doctors, and a change in health care system rules that is necessary. Moreover, we have to be aware that stereotypes about patients with MUS are not helpful, as this patient group is very heterogeneous. It is only a small subgroup of patients with MUS who are the really "difficult to treat" patients. Many patients with MUS are open for doctors' alternative explanations about the origins of their symptoms, and can be motivated to cope with the symptoms. Others have comorbid psychiatric disorders such as depression or panic disorder, which should be treated adequately. These complex issues mean that guidelines for physicians that cover medical decisions (e.g., which investigations and treatments are recommended for which subgroup of patients with MUS) and behavioral recommendations are an important future development in this field.

Acknowledgement: I am grateful to all collaborating general practitioners and internal specialists in Marburg (Germany) who helped me to get insight in their daily work.

Corresponding Author: Winfried Rief, PhD; Clinical Psychology and Psychotherapy, Philipps University of Marburg, Gutenbergstrasse 18, Marburg 35032, Germany (e-mail: rief@staff.uni-marburg.de). 


\section{REFERENCES}

1. Fink P, Rosendal M, Olesen F. Classification of somatization and functional somatic symptoms in primary care. Aust $\mathrm{N} \mathrm{Z} \mathrm{J} \mathrm{Psychiatry.}$ 2005;39:772-81.

2. Fink P, Toft T, Hansen MS, Ornbol E, Olesen F. Symptoms and syndromes of bodily distress. Psychosom Med. 2007;69(1):30-9.

3. Smith RC, Dwamena FC. Classification and diagnosis of patients with medically unexplained symptoms. J Gen Intern Med. DOI 10.1007/ s11606-006-0067-2 (in this issue).

4. Mayou R, Sharpe M, Kirmayer LJ, Simon G, Kroenke K. Somatoform disorders: time for a new approach in DSM-V. Am J Psychiatr. 2005;162:847-55.

5. Kroenke K. Physical symptom disorder: a simpler diagnostic category for somatization-spectrum conditions. J Psychosom Res. 2006;60:335-9.

6. Rief w, Hiller w. Toward empirically based criteria for somatoform disorders. J Psychosom Res. 1999;46:507-18.

7. Rief W, Henningsen P, Hiller W. Classification of somatoform disorders. Am J Psychiatr. 2006; 163:746-7.

8. McHugh PR. Striving for coherence. Psychiatry's efforts over classification. JAMA. 2005;293(20):2526-8.

9. Simon G, Ormel J, VonKorff M, Barlow W. Health care costs associated with depressive and anxiety disorders in primary care. Am J Psychiatr. 1995; 152:352-7.

10. Rief W, Martin A, Klaiberg A, Brähler E. Specific effects of depression, panic, and somatic symptoms on illness behavior. Psychosom Med. 2005;67:596-601.

11. Weich S, Lewis G, Donmall R, Mann A. Somatic presentation of psychiatric morbidity in general practice. Br J Gen Pract. 1995;45:143-7.

12. Rief W, Nanke A, Emmerich J, Bender A, Zech T. Causal illness attributions in somatoform disorders-associations with comorbidity and illness behavior. J Psychosom Res. 2004;57:367-71.

13. Rief W, Heitmüller AM, Reisberg $\mathbf{K}$, Rüddel $\mathbf{H}$. Why reassurance fails in patients with unexplained symptoms-an experimental investigation of remembered probabilities. PLoS Med. 2006;3(8):e269.

14. Barsky AJ, Orav J, Bates DW. Somatization increases medical utilization and costs independent of psychiatric and medical morbidity. Arch Gen Psychiatry. 2005;62:903-10.

15. Rief W, Martin A, Rauh E, Zech T, Bender A. Evaluation of a general practitioners' training: how to manage patients with unexplained physical symptoms. Psychosomatics. 2006;47:304-11.

16. Salmon P, Peters S, Clifford R, et al. Why do general practitioners decline training to improve management of medically unexplained symptoms? DOI 10.1007/s11606-006-0094-z (in this issue). 